\title{
EVALUACIÓN TÉRMICA DE MODIFICACIONES EN LAS ENVOLVENTES DE MÓDULOS EXPERIMENTALES UBICADOS EN EL CENTRO POBLADO DE IMATA (4519 MSNM), AREQUIPA
}

\author{
THERMAL EVALUATION OF THE MODIFICATIONS IN THE \\ ENVELOPES OF EXPERIMENTAL MODULES LOCATED IN THE \\ COMMUNITY OF IMATA (4519 MASL), AREQUIPA
}

\author{
Martin Ponce-Gonzales ${ }^{10}$, Juan Molina-Fuertes ${ }^{10}$ \\ Manfred Horn-Mutschler ${ }^{*}$ ([), Mónica Gómez-León ${ }^{1}$ (])
}

${ }^{1}$ Facultad de Ciencias, Universidad Nacional de Ingeniería, Lima, Perú

Recibido (Received): 01 / $03 / 2021$ Aceptado (Accepted): 18/06/2021

\begin{abstract}
RESUMEN
Este trabajo analiza los diferentes aportes térmicos y energéticos dinámicos de las diferentes propuestas de modificaciones realizados en las envolventes de dos módulos experimentales de vivienda construidos en el centro poblado de Imata, Arequipa a 4519 msnm. Los módulos fueron construidos con características constructivas del lugar y diseño típico, posteriormente se realizaron modificaciones como cambiar el material de cubierta del techo, enlucido de las paredes de adobe con yeso, colocación de claraboyas en el techo y falso techo, aislamiento del falso techo, y construcción de un invernadero adosado en uno de los módulos. El registro de datos consistió en medir la temperatura del aire interior de los módulos e invernadero adosado, además, del registro de datos meteorológicos exteriores, utilizando una estación Davis ubicada en lo alto de la municipalidad de Imata. La temperatura media diaria del aire interior del módulo sin invernadero $(\mathrm{M} 1)$ inicialmente fue de $3,1^{\circ} \mathrm{C}$, siendo la temperatura media diaria exterior $0,61^{\circ} \mathrm{C}$; con las modificaciones de las envolventes, la temperatura media del aire interior de $\mathrm{M} 1$ fue $10{ }^{\circ} \mathrm{C}$, aportando en promedio al interior $6,9^{\circ} \mathrm{C}$; para el módulo con invernadero $(\mathrm{M} 2)$, la temperatura media del aire interior fue de 14 , $6^{\circ} \mathrm{C}$, aportando en promedio al interior $11,5^{\circ} \mathrm{C}$ con una diferencia promedio de $5,17^{\circ} \mathrm{C}$ por encima de M1. Los resultados obtenidos demuestran el aporte de las modificaciones y técnicas bioclimáticas implementadas como el caso del invernadero adosado que para las zonas Altoandinas por su potencial solar se presentan idóneos y eficaces.
\end{abstract}

Palabras Clave: Energía Solar, Envolventes, Simulación térmica, Módulos experimentales.

\section{ABSTRACT}

This paper analyzes the different thermal and dynamic energy contributions of the different proposals for modifications made in the envelopes of two experimental housing modules built in the town of Imata, Arequipa at 4519 meters above sea level. The modules were built with constructive characteristics of the place and typical design, later modifications were made such as changing the roof covering material, plastering the adobe walls with plaster, placing skylights on the ceiling and false ceiling, insulation of the false ceiling, and construction of a greenhouse attached to one of the modules. The data recording consisted of measuring the indoor air temperature of the modules and attached greenhouse, in addition to the outdoor meteorological data recording using a Davis station located at the top of the Imata municipality. The average daily indoor air temperature of the module without greenhouse $(\mathrm{M} 1)$ was initially $3.1^{\circ} \mathrm{C}$, the average daily outdoor temperature being $0.61{ }^{\circ} \mathrm{C}$; With the modifications of the envelopes, the average temperature of the interior air of $\mathrm{M} 1$ was $10{ }^{\circ} \mathrm{C}$, contributing an average of $6.9^{\circ} \mathrm{C}$ to the interior; For the module with greenhouse $\left(\mathrm{M}_{2}\right)$, the mean indoor air temperature was $14.6{ }^{\circ} \mathrm{C}$, contributing an average of $11.5^{\circ} \mathrm{C}$ indoors with an average difference of $5.17{ }^{\circ} \mathrm{C}$ above $\mathrm{M} 1$. The results obtained demonstrate the contribution of the bioclimatic modifications and techniques implemented, such as the case of the attached greenhouse, which are suitable and effective for the High Andean areas due to their solar potential.

Keywords: Solar energy, Envelopes, Thermal simulation, Experimental modules.

1 Corresponding author.:

E-mail: mhorn@uni.edu.pe

1. INTRODUCCIÓN
En las zonas Altoandinas de Perú, con altitudes mayores a 4000 msnm, se dan climas muy particulares, 
con promedios de oscilaciones térmicas anuales mayores a $15{ }^{\circ} \mathrm{C}$, promedios de temperaturas mínimas bajo $0{ }^{\circ} \mathrm{C}$ situaciones conocidas como heladas y un potencial solar incidente promedio anual mayor a 6 $\mathrm{kWhm}^{-2} \mathrm{dí}^{-1}$ [1]. Todos los años estos fenómenos climatológicos ocurren entre los meses de abril a setiembre y se ven afectadas las poblaciones Altoandinas en alto riesgo (niños menores de 5 años y adultos mayores de 65 años), sea por la condición social de pobreza y pobreza extrema, la nutrición o ubicación geográfica, actividades como la agricultura, ganadería y educación, por esto el Gobierno Peruano ha priorizado la inclusión y protección de las poblaciones más vulnerables a estos fenómenos [2].

El 2016, la población en alto riesgo frente a las heladas fue más del 30\% según el Instituto Nacional de Estadísticas e Informática (INEI) [3], el 2017 hubo 312 personas fallecidas a causa de las bajas temperaturas, Instituto Nacional de Defensa Civil (INDECI) [4] y para la Defensoría del Pueblo el 2018 hubo 182 personas fallecidas por las heladas y friaje [5].

Las mayores ganancias de calor al interior de las viviendas es debida por la combinación de los efectos del clima y las características termo-físicas de los materiales que componen la envolvente [6], se considera también criterios bioclimáticos como la ubicación, orientación, distribución y geometría [7], estos factores contribuyen en obtener un ambiente saludable para alcanzar el bienestar térmico dentro de la vivienda. En las zonas Altoandinas la elección de los materiales no es la idónea, como es el caso de la "calamina metálica" que, por sus propiedades de buen conductor del calor no genera protección térmica, sin embargo, por a su bajo costo, fácil acceso, transporte e instalación, su uso se ha masificado.

En este aspecto, este trabajo aborda propuestas de modificaciones de la envolvente de dos módulos experimentales de viviendas Altoandinas con y sin invernadero adosado utilizando previamente la simulación térmica con EnergyPlus, para el análisis de su comportamiento térmico, siendo la evaluación del incremento de temperatura en el interior del módulo de acuerdo a diferentes configuraciones realizadas en diferentes períodos, el principal objetivo. Los datos se registraron utilizando una estación meteorológica Vantage DAVIS Pro Plus, datas logger y sensores de temperatura Pt10o. Finalmente, los resultados analíticos del modelamiento y simulación de los módulos utilizando EnergyPlus fueron validados con la experimentación tomando como referencia la temperatura del aire interior.

\section{ANTECEDENTES}

Desde el 2009 en la Universidad Nacional de Ingeniería (UNI), la Facultad de Ciencias y el Centro de Energías Renovables (CER-UNI), se desarrollan trabajos de investigación relacionado al diseño Bioclimático y bienestar térmico, enfocados en mejorar las condiciones térmicas de habitabilidad de las poblaciones Altoandinas con el objetivo de lograr incrementos de la temperatura del aire interior a partir de la evaluación y análisis de prototipos de viviendas y módulos experimentales construidos in situ, a alturas , mayores de los $3500 \mathrm{msnm}$ priorizando el aprovechamiento del potencial solar de dichas zonas. Varios de estos estudios en su etapa de diseño se apoyan en la modelación y simulación térmicaenergética utilizando el EnergyPlus y sus complementos el SketchUp y OpenStudio.

El 2014 el Ministerio de Vivienda Construcción y Saneamiento (MVCS), promulgó la Norma Técnica Peruana EM.110 "Confort Térmico y Lumínico con Eficiencia Energética" [8], refiriendo su aplicación voluntaria a las nuevas construcciones y la remodelación, refacción y acondicionamiento de edificaciones existentes según la ley establecida 29090, para el ámbito rural y urbano del país. La norma establece parámetros técnicos de diseño para el confort térmico y lumínico de acuerdo a criterios bioclimáticos para la construcción; su elaboración ha sido adaptada al medio local tomando como referencias normas extranjeras de Argentina, México y España. Se dispone que la zonificación bioclimática del Perú consiste en nueve zonas e infiere para cada uno de ellas el cumplimiento de transmitancias térmicas máximas para piso, paredes, y techo, de no ser así, el proyecto será considerado no viable. El mismo año se publicó el libro "Abrigando hogares" del MVCS-GIZ cuya finalidad es que los pobladores rurales adopten determinadas técnicas y puedan replicarlas en su vivienda como el restaurar y mejorar su envolvente con materiales de la zona; estas actividades engloban a las autoridades locales, regionales y gobierno central que conjuntamente con la academia y la población, se alcancen los objetivos necesarios de mejorar la calidad de vida del poblador Altoandino [9].

El estudio precedente de los módulos experimentales de vivienda fue el trabajo realizado en el año 2018 [10], donde, se realizó el estudio de 5 configuraciones de módulos diferentes utilizando EnergyPlus, añadiendo diferentes componentes bioclimáticos al diseño inicial. Se realizaron simulaciones para obtener los diferentes comportamientos óptimos de temperatura, energía e intercambios de aire, a partir de data del clima para Imata de un año típico meteorológico generado con el programa Meteonorm. Dos de las configuraciones de 
módulos se construyeron el mismo año de su estudio, la cual sirvió de base para el presente estudio. Otro precedente más actual es el trabajo presentado al congreso mundial de energía solar de la ISES (International Solar Energy Society), desarrollado en Chile (2019), referente al estudio de evaluación por comparación y validación del aporte térmico del invernadero adosado para calentar el aire interior del módulo experimental [11].

\subsection{DETALLES DE LOS EXPERIMENTALES (M1 Y M2)}

MÓDULOS

Los módulos de vivienda experimentales sin invernadero (M1) y con invernadero (M2) fueron construidos en el centro poblado de Imata a $4519 \mathrm{msnm}$ (latitud de $15^{\circ} 50$ ' 18,92" Sur y longitud de $71^{\circ} 5$ ' 28,46" Oeste), distrito de San Antonio de Chuca, provincia de Caylloma, región Arequipa, a una, al sur del Perú. Los módulos tienen las mismas dimensiones interiores de 3 $\mathrm{m} \times 4 \mathrm{~m} \times 2,3 \mathrm{~m}$, con una superficie exterior de 18,24 $\mathrm{m}^{2}$ por cada módulo; los componentes constructivos de cada módulo son los mismos con la diferencia que uno lleva un invernadero adosado, las paredes son de adobe enlucidos por el interior y exterior con yeso, el piso es de tierra, la puerta lleva marco de madera y hoja de calamina metálica, las ventanas son de marco de metal y vidrio simple ( $3 \mathrm{~mm}$ ), techo de fibrocemento de $4 \mathrm{~mm}$, falso techo de triplay de $4 \mathrm{~mm}$ con poliestireno expandido de $0,05 \mathrm{~m}$ de espesor, claraboyas de policarbonato alveolar de $6 \mathrm{~mm}$ con filtro UV de 3,12 a ambos lados de las alas del techo y falso techo los cuales conforman un solo sistema de ganancia de calor solar directa, y un invernadero adosado a $\mathrm{M} 2$ sobre su pared norte, ver figura 1 , adicional al falso se colocó poliestireno expandido detrás de la puerta metálica para eliminar el puente térmico, el ático cuenta con tres espacios de aire, una central que contiene las claraboyas del techo y falso techo, y dos laterales a la misma. El invernadero adosado a $M 2$ es de un ala y de base rectangular de 4,8 $\mathrm{m} \times 2 \mathrm{~m}$, y altura de 1,65 $\mathrm{m}$ en la parte baja y $2.2 \mathrm{~m}$ en la parte alta, lleva una puerta de marco de madera y hoja de policarbonato de 1,26 $\mathrm{m}^{2}$ la cual a la vez es la puerta de ingreso principal a M2. El material de cubierta del invernadero es de policarbonato alveolar cuya área traslucida es de 18,84 $\mathrm{m}^{2}$, el piso de concreto de 0,10 $\mathrm{m}$ de espesor, y la altura del muro lateral que lo conforma es de $0,85 \mathrm{~m}$, de adobe y enlucido con yeso $1,5 \mathrm{~cm}$ de espesor.

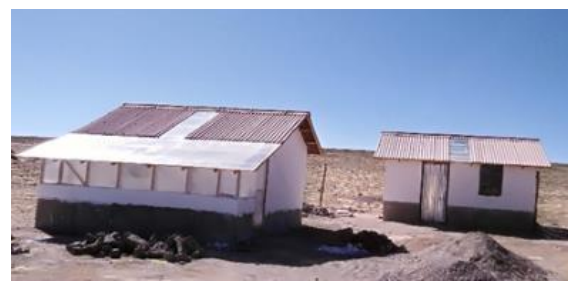

Fig. 1. Módulos experimentales de vivienda, M1 sin invernadero (atrás), M2 con invernadero (adelante).

\section{METODOLOGÍA}

La metodología comprende el análisis del estado inicial del módulo experimental denominado módulo típico, al cual posteriormente se le va adicionando en diferentes tiempos configuraciones de mejoras en su envolvente, tanto en paredes y techos, como el incluir un invernadero adosado a su pared norte y claraboyas en el techo El invernadero contribuirá en la mejora del incremento de temperatura del aire interior del módulo típico. Para cada configuración de modificación realizada se determina su aporte térmico y energético utilizando simulación dinámica.

El procedimiento para la evaluación y análisis térmico de dos módulos de vivienda experimentales $M_{1}$ y $\mathrm{M}_{2}$, referente a la parte energética, se inicia con el modelamiento en 3D de ambos módulos utilizando los programas SketchUp y OpenStudio, que son los complementos del programa de simulación EnergyPlus, creando las zonas térmicas y configurando las superficies de cada componente constructivo para finalmente crear el archivo de la arquitectura de los módulos con extensión IDF de entrada al programa EnergyPlus. Asimismo, modificando una plantilla del programa Elements a partir del ingreso de variables meteorológicas registradas, se generó un archivo de clima EPW de entrada al EnergyPlus, estos dos archivos son la base para el inicio de la simulación dinámica utilizando EnergyPlus [12]. La data meteorológica se registró con una estación Davis Vantage Pro Plus, instalada en la azotea de la municipalidad. Adicionalmente para las fechas en la cual la estación no registró datos, se consideró los datos generados por el programa Solcast, y el registro de datos in situ del Servicio Nacional de Meteorología e Hidrología del Perú (SENAMHI). El EnergyPlus es un programa de simulación energética de código abierto y uso gratuito, de propiedad del Departamento de Energía de los Estados Unidos, su entorno se basa en el desarrollo de modelos físico-matemáticos de balances energéticos que involucran flujos de masa y calor en estado dinámico, siendo el cálculo de la temperatura interior una de las variables de salida más solicitadas para el análisis del rendimiento térmico de edificios, además, del cálculo de las demandadas de energía de calefacción y refrigeración de un edificio. Su metodología de trabajo es por ensayo y error. Sus resultados tienen que ser validados con la parte experimental para posteriormente extrapolar los resultados a otras escalas de tiempo y análisis, e incluso realizar modificaciones respecto a adicionar o cambiar algún componente constructivo. En la tabla I se muestra el campo de entrada de las variables para las propiedades termo-físicas de los materiales utilizados en la 
construcción de los módulos, así como, valores de las propiedades ópticas para la simulación en EnergyPlus.

TABLA I

Propiedades termo-físicas y ópticas de los materiales para la simulación.

\begin{tabular}{cccccc}
\hline Materiales & $\begin{array}{c}\mathrm{k} \\
\left(\mathrm{Wm}^{-1} \mathrm{~K}^{-1}\right)\end{array}$ & $\begin{array}{c}\rho \\
\left(\mathrm{kgm}^{-3}\right)\end{array}$ & $\begin{array}{c}\mathrm{Ce} \\
\left(\mathrm{Jkg}^{-1} \mathrm{~K}^{-1}\right)\end{array}$ & $\varepsilon$ & $\alpha$ \\
\hline $\begin{array}{c}\text { Adobe } \\
\text { Calamina }\end{array}$ & 0,349 & 1600 & 920 & 0,92 & 0,65 \\
Tierra & 237 & 2702 & 903 & 0,03 & 0,09 \\
\hline
\end{tabular}

Nota: k: conductividad térmica, $\rho$ : densidad, Ce: calor específico, $\varepsilon$ : emitancia, $\alpha$ : absortancia solar.

El registro de datos de temperatura del aire interior se realizó con sensores de temperatura Pt100 conectados a Datas loggers Onset Hobo de cuatro canales. También se utilizó Micro estaciones de la misma marca ubicándose aproximadamente en el centro geométrico del interior del módulo. Los módulos inicialmente fueron construidos con características similares en su envolvente, asemejándose a una vivienda de construcción típica actual de Imata, es decir, con paredes de adobe, techo de calamina metálica, ventana de un solo vidrio con un marco de metal, y puerta de calamina metálica con marcos de madera. A partir de este diseño inicial, paulatinamente se realizaron cambios en la envolvente de los módulos experimentales $M_{1}$ y $M_{2}$, siendo el primer cambió el techo, utilizando fibrocemento y policarbonato para la claraboya, se consideró un falso techo con una parte transparente y otra opaca, en la primera se utiliza policarbonato, y en la segunda triplay sobre el cual lleva poliestireno expandido; el cambio se realizó primero en el $M 1$, mientras que el $M 2$ no contenía el poliestireno expandido, siendo esta la única diferencia con M1, del registro datos y mediciones, se determina el aporte térmico del material aislante. En otra configuración se tiene el invernadero adosado a $\mathrm{M} 2$ en su pared norte, mientras que $\mathrm{M} 1$ no cuenta con invernadero, para los intercambios de aire entre el invernadero y el interior de $M 2$, se realizaron algunas configuraciones de medida para diferentes estados de la puerta y la ventana que colindan con el invernadero, ambas abiertas o cerradas por completo; y ambas abiertas de 8:00 a 17:00 horas. Estas configuraciones se disponen como variables de entrada del EnergyPlus, así como las propiedades termofísicas de los materiales usados durante todas las configuraciones de las envolventes de los módulos, como el calor específico $\left(\mathrm{Jkg}^{-1} \mathrm{~K}^{-1}\right)$, la conductividad térmica $\left(\mathrm{Wm}^{-1} \mathrm{~K}^{-1}\right)$ y la densidad $\left(\mathrm{kgm}^{-3}\right)$. Para los valores de estos parámetros se tomaron de la Norma Técnica Peruana EM.110 "Confort térmico y lumínico con eficiencia energética", resaltando la importancia de cumplir con las transmitancias térmicas máximas en el techo, paredes y piso Se simularon las configuraciones de los módulos experimentales, todas basadas en mejoras de aislamiento, captación de energía solar, y reducción de infiltraciones, de acuerdo con las técnicas consideradas durante el proceso de cambios en la envolvente con la finalidad de alcanzar un óptimo bienestar térmico interior.

La tabla II muestra los períodos de mediciones de los módulos experimentales de acuerdo a los cambios realizados en las envolventes. En la etapa inicial se representa al módulo típico con techo de calamina metálica, puerta con marco de madera y hoja de calamina metálica, así como las paredes de adobe (M1T). En el período intermedio el módulo inicial presenta cambios en el techo, ahora es de fibrocemento y lleva claraboya de policarbonato alveolar, la puerta es de marco de madera y hoja de calamina metálica, las paredes son de adobe con enlucido de yeso en su interior, y el falso techo sobre la superficie opaca lleva poliestireno expandido (M1FP); en tanto que el segundo módulo presenta las mismas características del módulo descrito anteriormente pero sin la inclusión del poliestireno expandido (M2FSP), y el módulo con invernadero adosado en su pared norte representa el módulo 2 (M2l).

TABLA II

Etapas de medidas en los módulos experimentales.

\begin{tabular}{ccc}
\hline Estados & $\begin{array}{c}\text { Modificaciones } \\
\text { en las } \\
\text { envolventes }\end{array}$ & Fecha \\
\hline Inicial & M1T & $\begin{array}{c}26-31 \\
\text { jul. }\end{array}$ \\
\hline Intermedio & M1FP, M2FSP & $\begin{array}{c}31-\text { oct. } \\
\text { o6-nov. }\end{array}$ \\
\hline Final & M2I & $\begin{array}{c}\text { 02-06 } \\
\text { dic. }\end{array}$
\end{tabular}

Nota: Por cada configuración efectuada se evaluó el incremento de la temperatura al interior del módulo.

La figura 3 muestra los instrumentos utilizados en la medición y registro de datos de temperatura del aire al interior del módulo y del invernadero, así como, la estación meteorológica Davis Vantage Pro Plus. Los datos se registraron cada 15 minutos como, temperatura del aire $\left({ }^{\circ} \mathrm{C}\right)$, irradiancia solar $\left(\mathrm{Wm}^{-2}\right)$, humedad relativa (\%), dirección $\left({ }^{\circ}\right.$ ) y velocidad del viento $\left(\mathrm{ms}^{-1}\right)$. La letra (a) muestra el data logger Hobo (registrador de datos de 4 canales), la letra (b) muestra la estación meteorológica modelo Davis Vantage Pro Plus instalada en el cuarto nivel de la municipalidad de Imata y la letra (c) muestra una especie de cono hacia abajo y envuelto con papel reflectante el cual alberga un sensor de temperatura de aire Pt10o en su interior, con la finalidad que no le llegue la radiación solar y altere las mediciones.

Siendo el parámetro principal de análisis la temperatura en el interior de los módulos experimentales de vivienda, en la simulación y validación de la misma no se tomó en cuenta la simulación del invernadero para el estudio. 
M. Ponce et al.

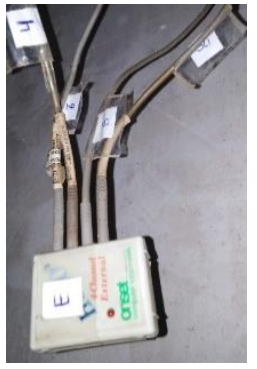

(a)

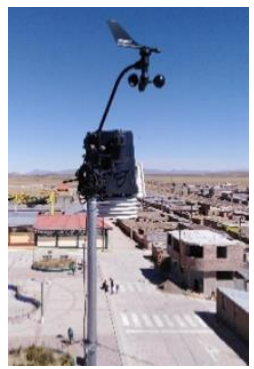

(b)

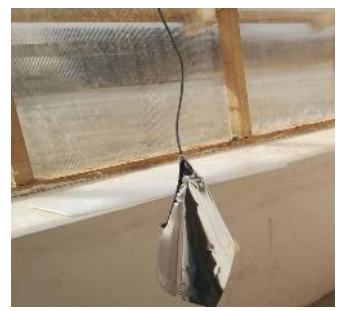

(c)

Fig. 3. Dispositivos de medidas. (a) Registrador de datos de temperatura, (b) Estación meteorológica, (c) Sensor de temperatura Pt100 protegido de la radiación solar.

\section{ANÁLISIS DE RESULTADOS}

Se presenta el análisis de los resultados de las etapas de medida según metodología de experimentación por comparación de los módulos experimentales. Se valida los datos analíticos de la simulación con data experimental in situ mediante comparación gráfica obteniendo buenos acuerdos entre lo medido y simulado. También se presenta el análisis de las condiciones meteorológicas externas para Imata según data del SENAMHI que cuenta con una estación en el propio lugar de parámetros como, la temperatura del aire, la humedad relativa, y la dirección y velocidad del viento. La irradiancia solar se analiza con data del programa Solcast.

\subsection{TEMPERTAURA DEL AIRE EXTERIOR}

La figura 4 resume los valores de la temperatura del aire promedio mensual correspondiente al año 2018 para el centro poblado de Imata, datos registrados por el SENAMHI que cuenta con una estación ubicada en el lugar de estudio [13]. Se muestra que desde el mes de mayo hasta setiembre la temperatura media presenta valores por debajo a $2^{\circ} \mathrm{C}$, siendo estos meses de heladas o bajas temperaturas, intensificándose en junio y julio, época de estiaje.

La figura 5 muestra la irradiación solar promedio mensual para un año (2018) en Imata. Los valores analizados corresponden a la empresa de servicios de datos solares Solcast, que proporciona datos de energía e irradiancia solar reales y pronosticados, a nivel mundial, utilizando satélites y mediciones de superficie [14], esta data se utilizó dada la carencia de datos in situ de este parámetro. Para asegurar el uso de la data del Solcast, esta se comparó con data registrada por la estación Davis que registra irradiancia solar global sobre superficie horizontal, resultando diferencias promedio no mayor de $8 \mathrm{Wm}^{-2}$, esto asegura una buena aproximación y representación de la incidencia solar en el propio lugar, por tal motivo, esta misma data se utilizó como parte de los parámetros meteorológicos requeridos para generar un archivo de clima EPW de entrada al EnergyPlus. Con estas consideraciones se representó la irradiación solar anual, promedio mensual de Imata, observándose que para el mes de noviembre el promedio supera los $7 \mathrm{kWhm}^{-2} \mathrm{día}^{-1}$, con mínimos de 4,64 kWhm ${ }^{-2} \mathrm{dí}^{-1}$ para el mes de junio.

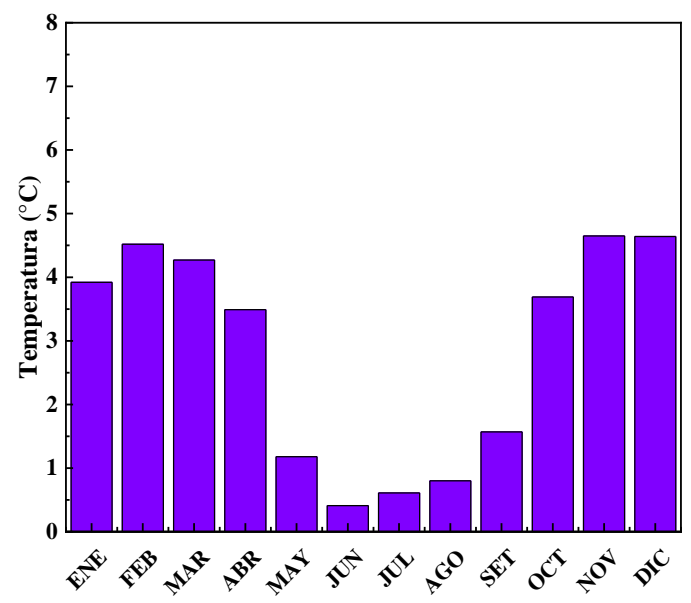

Fig. 4. Temperatura media mensual de Imata según datos del SENAMHI para el año 2018.

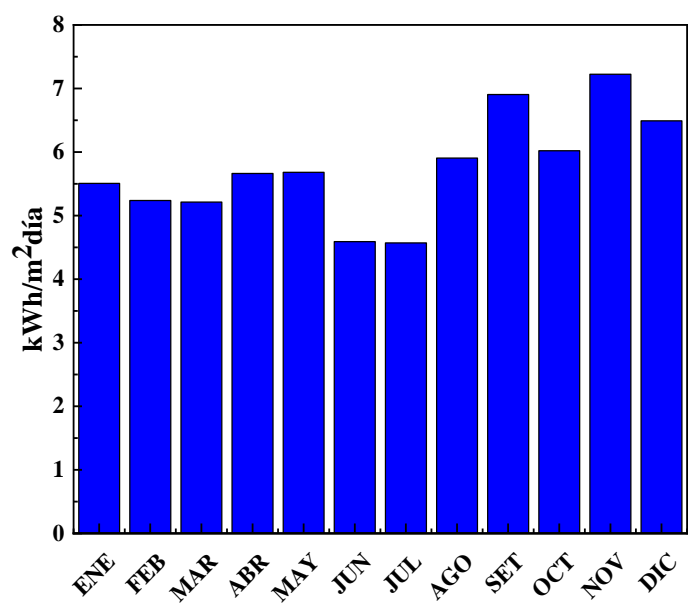

Fig. 5. Irradiación solar media diaria mensual $\left(\mathrm{kWhm}^{-2} \mathrm{dí}^{-1}\right)$ para Imata según archivo de Solcast para el año 2018.

La Tabla III resume los valores mensuales de temperaturas del año 2018, se muestra la temperatura diaria mensual, la media ( $(\mathrm{m})$, media máxima (Tmáxm), y media mínima (Tmínm); temperatura extrema mensual, máxima y mínima, donde, el mes que registra la temperatura más baja es julio, con una media de 1,2 ${ }^{\circ} \mathrm{C}$, y temperaturas extremas mínimas de hasta $-15,3{ }^{\circ} \mathrm{C}$ para el mes de setiembre, mientras que el mes de 
M. Ponce et al.

diciembre presenta las temperaturas más altas con una media de $5,3{ }^{\circ} \mathrm{C}$, y temperaturas extremas diarias mensuales de $-10,9{ }^{\circ} \mathrm{C}$ y $17,5{ }^{\circ} \mathrm{C}$. La oscilación térmica promedio anual fue de $16,2{ }^{\circ} \mathrm{C}$. La temperatura media anual fue de $3,7{ }^{\circ} \mathrm{C}$, las mínimas temperaturas se registraron entre las 4:00 y 5:30 horas del día y las máximas entre las 12:00 y 15:00 horas del día. Respecto a las temperaturas mínimas extremas e incluso temperaturas medias mínimas, la problemática de las heladas para Imata se presentan durante todo el año y no solo en los meses de mayo a setiembre. Intensificándose en este caso para el año 2018, en los meses de agosto a setiembre y las temperaturas máximas no superan los $18^{\circ} \mathrm{C}$.

TABLA III

Temperatura del aire exterior para el centro poblado de Imata.

\begin{tabular}{cccccccccccccc}
\hline Mes & ENE & FEB & MAR & ABR & MAY & JUN & JUL & AGO & SET & OCT & NOV & DIC & Prom. \\
\hline Tmáxm $\left({ }^{\circ} \mathrm{C}\right)$ & 10,8 & 11,2 & 11,0 & 12,4 & 11,5 & 9,2 & 9,2 & 10,7 & 13 & 13,2 & 15,3 & 13,9 & 11,8 \\
Tmínm $\left({ }^{\circ} \mathrm{C}\right)$ & $-0,5$ & 0,6 & 0,2 & $-3,0$ & $-7,4$ & $-6,3$ & $-6,8$ & $-8,0$ & $-9,4$ & $-4,5$ & $-4,8$ & $-3,3$ & $-4,4$ \\
Tm $\left({ }^{\circ} \mathrm{C}\right)$ & 5,1 & 5,9 & 5,6 & 4,7 & 2,1 & 1,4 & 1,2 & 1,4 & 1,8 & 4,4 & 5,3 & 5,3 & 3,7 \\
Temp. Máx. & 13,9 & 13,9 & 13,3 & 14,5 & 14,3 & 11,7 & 13,7 & 12,4 & 15,5 & 16,1 & 17,1 & 17,5 & 14,5 \\
Extrema $\left({ }^{\circ} \mathrm{C}\right)$ & & & & & & & & & & & & & \\
Temp. Mín. & $-3,9$ & $-3,9$ & $-4,8$ & $-6,0$ & $-13,2$ & $-13,1$ & $-11,1$ & $-13,5$ & $-15,3$ & $-9,5$ & $-9,3$ & $-10,9$ & $-9,5$ \\
Extrema $\left({ }^{\circ} \mathrm{C}\right)$ & & & & & & & & & & & & & \\
Oscilación & 11,3 & 10,7 & 10,9 & 15,4 & 18,8 & 15,5 & 16,0 & 18,7 & 22,4 & 17,7 & 20,0 & 17,1 & 16,2 \\
Térmica $\left({ }^{\circ} \mathrm{C}\right)$ & $1,4,0$ \\
\hline
\end{tabular}

Nota: Temperaturas medias diarias ( $T m$ ), temperatura promedio máximo (Tmáxm), temperatura promedio mínimo diario (Tmínm), temperatura máxima extrema (Temp. Máx. Extrema) y temperatura mínima extrema (Temp. Mín. Extrema) mensuales para el año 2018.

\subsection{COMPARACIÓN TÉRMICA DE M1 Y M2}

La figura 6 muestra el comportamiento de la temperatura del aire interior ( $T_{\text {Int }}$ ) de $M 1$ (en todas las figuras, línea sólida azul), y la temperatura exterior ( $\mathrm{T}_{\mathrm{Ext}}$, desde ahora línea sólida verde), en su diseño inicial, denominado módulo típico. Se muestra en la imagen (figura 6a), la forma y disposición de los materiales empleados como, paredes de adobe, techo de calamina, puerta con marco de madera y hoja de calamina metálica, y ventana de vidrio simple con marco metálico. La figura $6 \mathrm{~b}$ corresponde a la etapa de medida del 26 al 31 de julio, donde, en promedio diario la temperatura del aire interior fue de $3,29{ }^{\circ} \mathrm{C}$, con una temperatura promedio del aire exterior de $1,91{ }^{\circ} \mathrm{C}$; presentándose en promedio, entre las 9:00 y 18:00 horas, una temperatura del aire interior, inferior al exterior en $2,5{ }^{\circ} \mathrm{C}$. La diferencia más notoria se da durante las 24:00 y 8:00 horas con una diferencia promedio de temperatura de $5,3{ }^{\circ} \mathrm{C}$, de ocurrencia alrededor de las 5:00 horas, la envolvente del módulo amortigua en promedio $3,5^{\circ} \mathrm{C}$ de la temperatura interior con respecto de la exterior para el máximo que se dio entre las 14:30 y 15:30 horas para esos días de medición, mientras que para el mínimo la envolvente amortigua en $5,6{ }^{\circ} \mathrm{C}$ con respecto a la temperatura en el exterior, esto ocurrió entre las 5:00 y 6:30 horas.

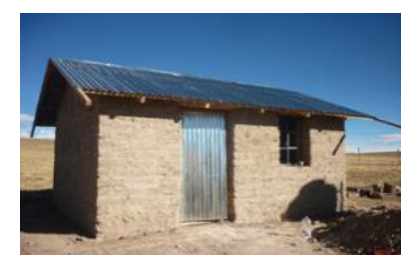

(a)

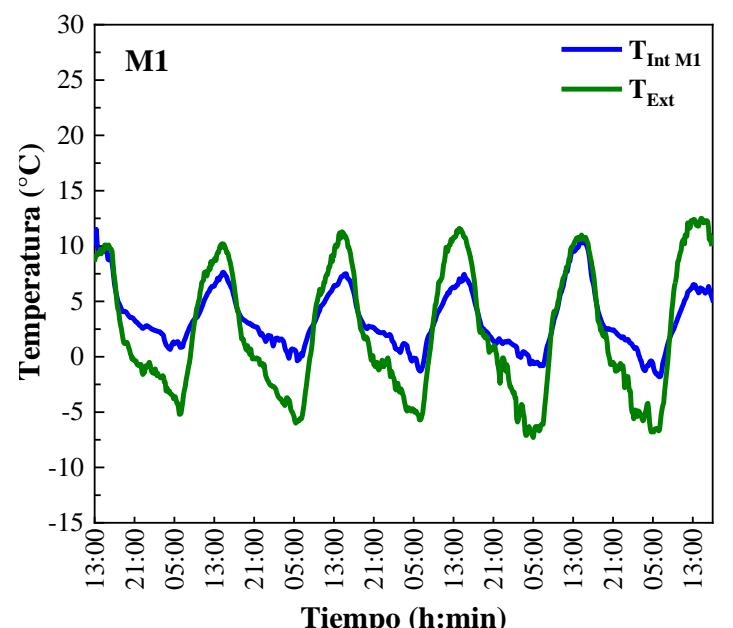

(b)

Fig. 6. Imagen del M1T y su comportamiento térmico. (a) M1T en su estado inicial. (b) Temperatura horaria en el interior del M1, TInt M1 (línea sólida azul), temperatura del aire exterior $\mathrm{T}_{\text {Ext }}$ (línea sólida verde).

La figura 7 muestra el comportamiento de la temperatura del aire interior de $M_{1}\left(T_{I n t M 1}\right)$ y $M_{2}\left(T_{I n t M 2}\right.$, desde ahora línea sólida anaranjada), y la temperatura exterior $\left(T_{E x t}\right)$. La figura 7a muestra el interior del ático de M1 cuya base lleva poliestireno expandido sobre 
tableros de triplay configurando el falso techo, y la figura $7 \mathrm{~b}$ una vista del falso techo del interior del módulo, mientras que $\mathrm{M}_{2}$ cuenta con falso techo solo de triplay. Para este caso el registro de medida fue del 31 de octubre al 3 de noviembre (figura $7 c$ ), donde, en promedio diario la temperatura del aire interior para $\mathrm{M} 1$ fue de $8,73{ }^{\circ} \mathrm{C}$, y para $\mathrm{M}_{2} 8,06{ }^{\circ} \mathrm{C}$, siendo mayor la temperatura de $\mathrm{M} 2$ en $0,67{ }^{\circ} \mathrm{C}$ con respecto a $\mathrm{M} 1$, esta diferencia puede inferirse que es el aporte del poliestireno expandido de 0,05 $\mathrm{m}$ de espesor, con una temperatura promedio del aire exterior de $4,64{ }^{\circ} \mathrm{C}$; presentándose en promedio entre las 9:00 y 18:00 horas una temperatura en el aire interior, superior a la exterior en $2,17{ }^{\circ} \mathrm{C}$ para $\mathrm{M}_{1}$, y $2,04{ }^{\circ} \mathrm{C}$ para $\mathrm{M}_{2}$, la diferencia más relevante se da entre las 3:00 y 6:00 horas con una diferencia promedio de temperatura de $7,8^{\circ} \mathrm{C}$ para $\mathrm{M} 1$, y $6,46{ }^{\circ} \mathrm{C}$ para $\mathrm{M} 2$, de ocurrencia alrededor de las 5:00 horas.

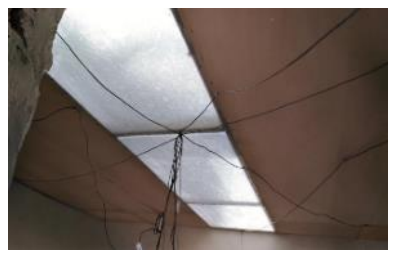

(a)

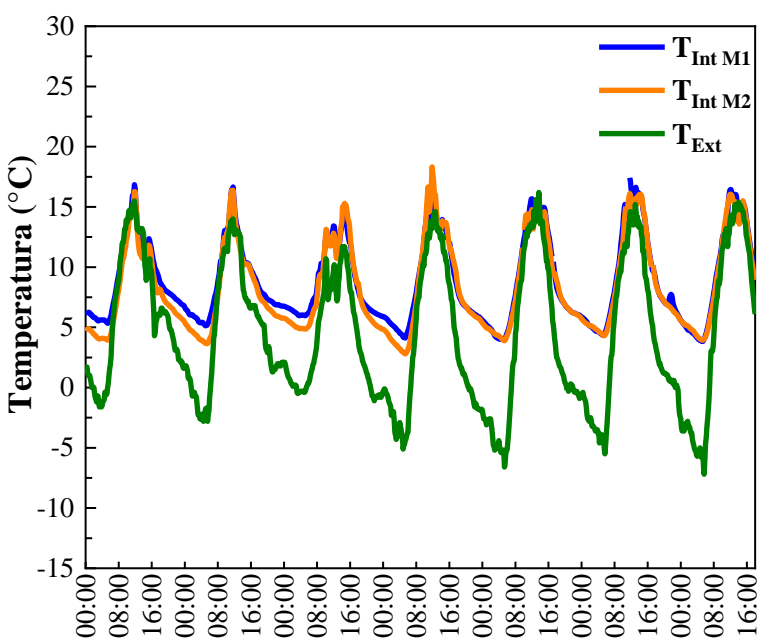

Tiempo (h:min)

(c)

Fig. 7. Falso techo de los módulos y su comportamiento térmico.

(a) Falso techo con divisiones de triplay y policarbonato, (b)

Poliestireno expandido sobre falso techo, (c) Temperatura horaria

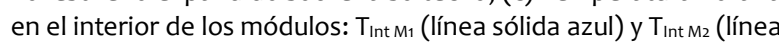
sólida anaranjada), temperatura exterior $\mathrm{T}_{\text {Ext }}$ (línea sólida verde), del 31 de octubre al 06 de noviembre.

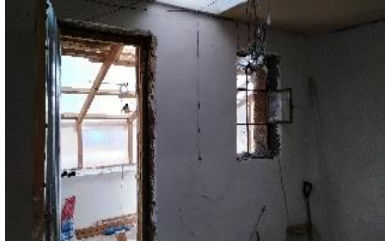

(a)

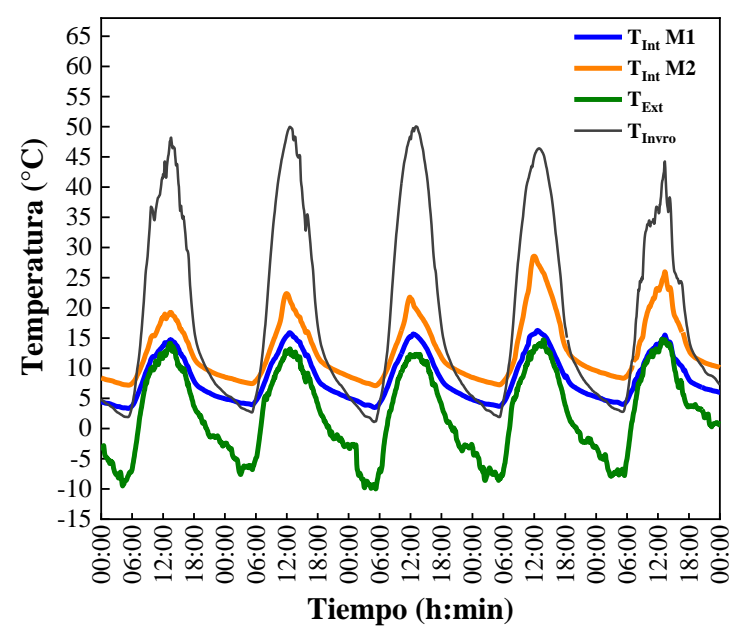

(c)

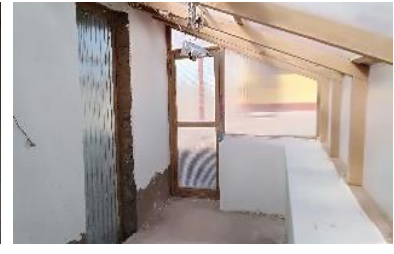

(b)
Fig. 8. Interior del invernadero y comportamiento térmico de invernadero y de los módulos. (a) Puerta y ventana que comunican el módulo y el invernadero, (b) ambiente del invernadero, (c) Temperatura horaria en el interior de los módulos, $T_{\text {Int } M_{1}}$ (línea sólida azul) y $\mathrm{T}_{\text {Int M2 }}$ (línea sólida anaranjada), temperatura del invernadero

$\mathrm{T}_{\text {Invro }}$ (línea sólida negra), temperatura exterior $\mathrm{T}_{\text {Ext }}$ (línea sólida verde), del 02 al 06 de diciembre.

La figura 8 muestra el comportamiento de la temperatura del aire interior de $M_{1}\left(T_{\operatorname{lnt} M_{1}}\right)$ y $M_{2}\left(T_{\operatorname{lnt} M_{2}}\right)$, la temperatura exterior $\left(T_{E x t}\right)$, y la temperatura del aire en el invernadero. La figura 8a muestra parte del interior de $M_{2}$, que cuenta con el invernadero adosado en su pared norte (figura 8b). La data de esta modificación corresponde del 2 al 4 de diciembre (figura $8 \mathrm{c}$ ), donde, en promedio diario la temperatura del ambiente interior de $\mathrm{M}_{1}$ fue de $8,2^{\circ} \mathrm{C}$, y de $\mathrm{M}_{2} 12,02{ }^{\circ} \mathrm{C}$, siendo la temperatura del aire de $\mathrm{M}_{2}$ mayor en $3,82{ }^{\circ} \mathrm{C}$ que $\mathrm{M} 1$, con temperatura promedio del aire exterior de $4,7{ }^{\circ} \mathrm{C}$; presentándose en promedio entre las 8:00 y 18:00 horas, una temperatura del aire interior para $M_{1}$ superior a la exterior en $2,9{ }^{\circ} \mathrm{C}$, y para $\mathrm{M} 2$ en $6,97^{\circ} \mathrm{C}$, la diferencia más notoria se da entre las 3:00 y 6:00 horas con una diferencia promedio de temperatura de $12,78^{\circ} \mathrm{C}$ para $\mathrm{M}_{1}$, y $16,45^{\circ} \mathrm{C}$ para el $\mathrm{M}_{2}$, estos valores promedios se dan aproximadamente cerca de las 5:00 horas. Del 5 al 6 de diciembre, en promedio diario, la temperatura del aire interior de $M 1$ fue $8,47{ }^{\circ} \mathrm{C}$, y para $\mathrm{M} 214,11^{\circ} \mathrm{C}$, de acuerdo a ello, se tiene que la temperatura del aire interior en $\mathrm{M}_{2}$ es mayor en $5,64{ }^{\circ} \mathrm{C}$ que $\mathrm{M}_{1}$, con una temperatura promedio del aire exterior de $2,89^{\circ} \mathrm{C}$. Para temperaturas promedio entre las 8:00 y 18:00 horas, el aire interior de $\mathrm{M} 1$ es superior al aire exterior en $2,13^{\circ} \mathrm{C}$, y para $\mathrm{M} 2$ en $9,92{ }^{\circ} \mathrm{C}$, la diferencia más notoria se da 
M. Ponce et al.

entre las 3:00 y 6:00 horas con una diferencia promedio de temperatura de $12,09{ }^{\circ} \mathrm{C}$ para $\mathrm{M} 1$ y $16,03{ }^{\circ} \mathrm{C}$ para $\mathrm{M}_{2}$, estos valores promedios se dan aproximadamente alrededor de las 5:00 horas, la temperatura máxima en el interior del invernadero en promedio diario fue de $47,1^{\circ} \mathrm{C}$, esto se dio entre las $13: 0$ y $14: 00$ horas, y la temperatura mínima fue de $2,1{ }^{\circ} \mathrm{C}$ en promedio diario dándose entre las 5:00 y 5:30 horas; en el cuarto y quinto día la temperatura en el interior del M2 aumenta tanto en los mínimos como en los máximos, esto es debido a que la puerta y la ventana que comunican el invernadero y la habitación del módulo están abiertas (ver figura 8a) en el horario de 8:00 a 17:00 horas.

\subsection{VALIDACIÓN DE LA SIMULACIÓN}

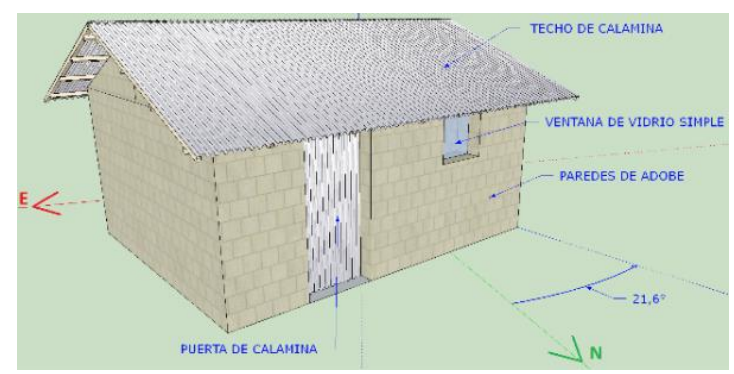

(a)

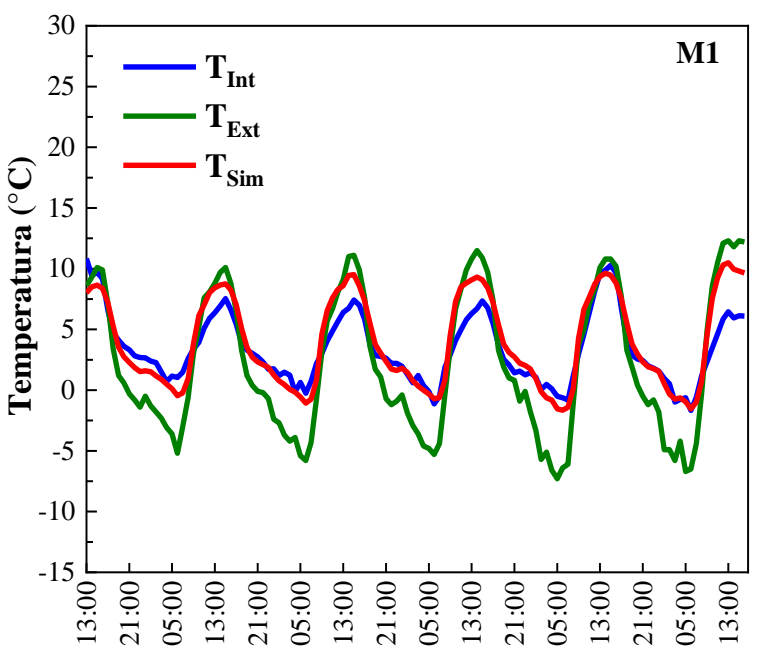

Tiempo (h:min)

(b)

Fig. 9. Imagen del M1 típico y su comportamiento térmico simulado.

(a) Representación del M1 en su estado inicial en SketchUp, (b) Temperatura horaria en el interior del módulo, $\mathrm{T}_{\text {Int }}$ (línea sólida azul), Temperatura simulada, $\mathrm{T}_{\text {Sim }}$ (línea sólida roja) y temperatura exterior $\mathrm{T}_{\text {Ext }}$ (línea sólida verde), del 26 al 31 de julio.

En la figura 9 se presentan los valores simulados y experimentales de la temperatura en el ambiente interior del módulo M1 (desde ahora línea azul para los valores experimentales y roja para la simulada) y la temperatura exterior (desde ahora línea verde sólida), inicialmente representa el módulo tradicional, para los días del 26 al 31 de julio, en promedio diario la temperatura experimental del ambiente interior fue de
$3,29{ }^{\circ} \mathrm{C}$ y la simulada $4,23{ }^{\circ} \mathrm{C}$, con una diferencia promedio diario de $0,94{ }^{\circ} \mathrm{C}$ entre lo experimental y lo simulado, con una temperatura promedio del aire exterior de $1,91{ }^{\circ} \mathrm{C}$. Para el módulo M1 se muestra la orientación con respecto al eje del norte con un ángulo de rotación de $21,6^{\circ}$ en dirección oeste.

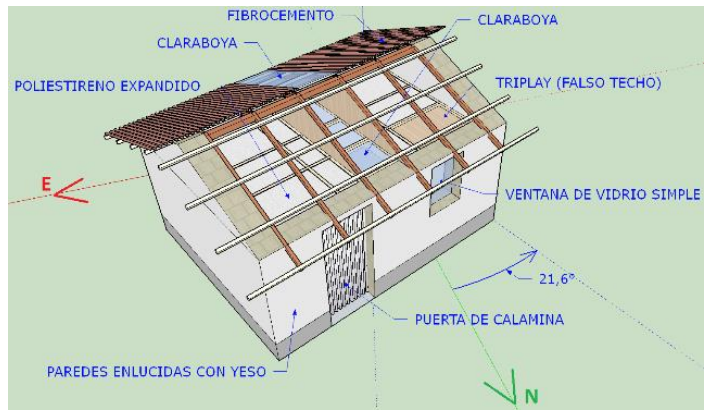

(a)

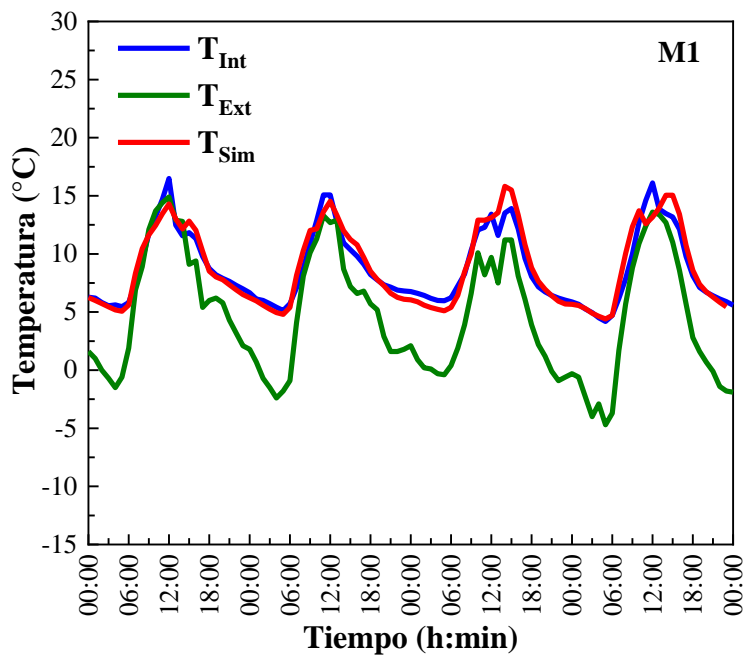

(b)

Fig. 10. Imagen del M1 mejorado y su comportamiento térmico simulado. (a) Representación del M1 en su estado intermedio en SketchUp, (b) Temperatura horaria en el interior del módulo, T Int

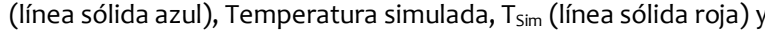
temperatura exterior $\mathrm{T}_{\text {Ext }}$ (línea sólida verde), del 31 de octubre al 03 de noviembre.

La figura 10 presenta los valores simulados y experimentales de la temperatura en el ambiente interior del módulo M1, la temperatura exterior, del 31 de octubre al 3 de noviembre, el módulo presenta en su configuración el falso techo con el poliestireno expandido incorporado sobre la base del falso techo, en promedio diario la temperatura experimental del ambiente interior fue de $8,75^{\circ} \mathrm{C}$ y la simulada $9,11^{\circ} \mathrm{C}$, con una diferencia promedio diario de $0,36{ }^{\circ} \mathrm{C}$ entre lo experimental y lo simulado, con una temperatura promedio del aire exterior de $4,65{ }^{\circ} \mathrm{C}$. El ángulo de rotación del $\mathrm{M} 1$ respecto del norte es $21,6^{\circ}$ en dirección oeste, observándose en la imagen (diseño en SketchUp) parte del ático con la construcción del falso techo y la incorporación del poliestireno expandido sobre la base de triplay. 
M. Ponce et al.

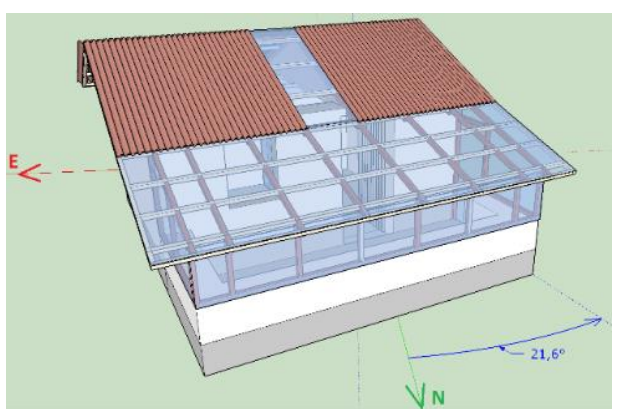

(a)

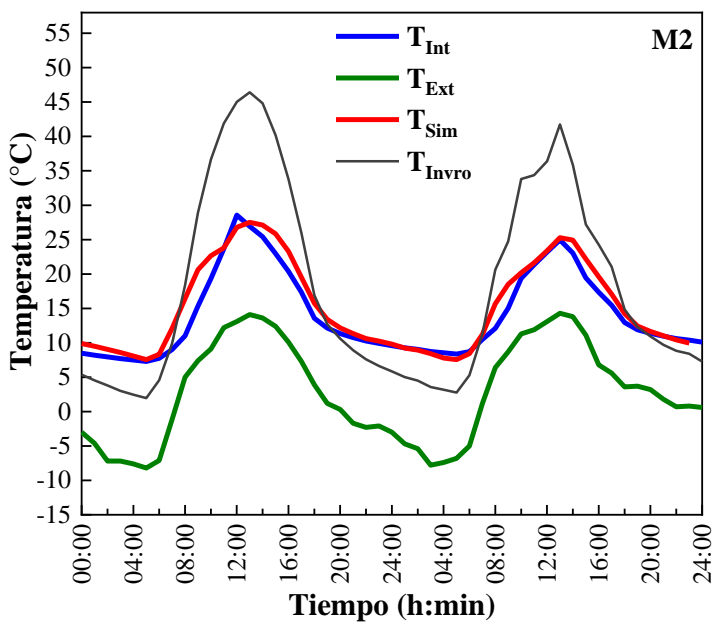

(b)

Fig. 11. Imagen del $\mathrm{M} 2$ con invernadero adosado y su comportamiento térmico simulado. (a) Representación del M2 en su estado final en SketchUp, (b) Temperatura horaria en el interior del módulo, $T_{\text {Int }}$ (línea sólida azul), Temperatura simulada, $T_{\text {sim }}$ (línea

sólida roja), temperatura exterior $\mathrm{T}_{\mathrm{Ext}}$ (línea sólida verde) y temperatura en el invernadero, $T_{\text {Invro }}$ (línea negra sólida), del 05 al 06 de diciembre.

En la figura 11 se presenta los valores simulados y experimentales de la temperatura en el ambiente interior del módulo $\mathrm{M}$ 2, la temperatura exterior y la temperatura en el ambiente interior del invernadero (línea negra sólida), del 5 al 6 de diciembre, con una configuración de puerta y ventana abiertas del $\mathrm{M} 2$ en el horario de 8:00 a 16:00 horas, en promedio diario la temperatura experimental del ambiente interior fue de $14,06{ }^{\circ} \mathrm{C}$ y la simulada $15,1{ }^{\circ} \mathrm{C}$, con una diferencia promedio diario de $1,1{ }^{\circ} \mathrm{C}$ entre lo experimental y lo simulado, con una temperatura promedio del aire exterior de $2,82{ }^{\circ} \mathrm{C}$ y temperatura promedio del aire interior del invernadero de $18,07{ }^{\circ} \mathrm{C}$. El ángulo de rotación del $\mathrm{M}_{2}$ y el invernadero adosado en su pared norte es de $21,6^{\circ}$ en dirección oeste, observándose en la imagen (diseño en SketchUp) tanto la puerta y la ventana del $\mathrm{M} 2$ abiertas.

\subsection{COMPARACIÓN ENERGÉTICA}

Se estimó la demanda energética de calefacción de acuerdo a las diferentes configuraciones y etapas de medida de los módulos experimentales, considerando mantener constante la temperatura del aire a $15{ }^{\circ} \mathrm{C}$ según el modelo adaptativo de Humphreys y Nicol que, con una temperatura promedio anual de $2,8^{\circ} \mathrm{C}$ y usando la data del SENAMHI se obtuvo para la temperatura neutra un valor de $15{ }^{\circ} \mathrm{C}$. Para la etapa del 26 al 31 de julio, con el diseño inicial de módulo típico para $\mathrm{M} 1$, la demanda de energía fue de 38,7 kWhdía ${ }^{-1}$, para la etapa del 31 de octubre al 3 de noviembre, con M1 ya mejorado con falso techo de triplay y sobre este poliestireno expandido, se obtuvo 13,5 $\mathrm{kWhdía}^{-1}$, mientras que, para $\mathrm{M} 2$ con falso techo pero sin poliestireno expandido, su demanda de energía fue de 20,8 kWhdía ${ }^{-1}$, obteniendo una diferencia de 7,3 kWhdía $^{-1}$ entre ambos módulos, valor que podría inferirse como ahorro de energía al mejorar el aislamiento utilizando poliestireno expandido. Para la etapa del 2 al 6 de diciembre se tiene configuraciones de puerta y ventana que comunican el interior de $\mathrm{M}_{2}$ con el interior del invernadero adosado, tal es así que, del 2 al 4 de diciembre las 24 horas se mantienen cerradas puerta y ventana de ambos módulos obteniéndose una demanda de 14,54 $\mathrm{kWhdía}^{-1}$ para M1 y 10,41 kWhdía-1 para M2; del 5 al 6 diciembre la puerta y ventana de $M 2$ permanecen abiertas de 8:00 a 17:00 horas, y en M1 permanecen cerradas las 24 horas, obteniéndose 14,23 kWhdía ${ }^{-1}$ para M1 y 10,29 kWhdía ${ }^{-1}$ para M2. La menor demanda de energía de calefacción se obtiene para la configuración de medida del 5 al 6 de diciembre con un ahorro de energía entre $M_{1}$ y $M_{2}$ de 3,94 kWhdía ${ }^{-1}$ debido al invernadero adosado sobre la pared norte de $\mathrm{M}_{2}$.

\section{CONCLUSIONES}

En promedio anual para el año 2018 la oscilación térmica de la temperatura del aire exterior fue de $16,2{ }^{\circ} \mathrm{C}$ con un máximo de $22,4{ }^{\circ} \mathrm{C}$ en el mes de setiembre y un mínimo de $10,7{ }^{\circ} \mathrm{C}$ en el mes de febrero. También se alcanzó temperaturas máximas extremas de $17,5{ }^{\circ} \mathrm{C}$ en el mes de diciembre y temperaturas mínimas extremas de hasta $-15,3{ }^{\circ} \mathrm{C}$ para el mes de setiembre. La energía solar media diaria que incide en Imata para el mismo año fue $5,75 \mathrm{kWhm}^{-2} \mathrm{día}^{-1}$, con un valor máximo de 7,24 kWhm ${ }^{-2} \mathrm{día}^{-1}$ en el mes de noviembre. Estos resultados muestran lo paradójico que es el clima de Imata, en el día existe abundante radiación solar y en las noches temperaturas bajo $0 \quad{ }^{\circ} \mathrm{C} \quad \mathrm{O}$ de heladas, intensificándose en los meses de abril a setiembre. En el proceso de la implementación de las diferentes técnicas bioclimáticas utilizadas en las modificaciones realizadas de las envolventes de M1 y M2, como, las mejoras del aislamiento térmico y uso de sistemas de climatización solar pasiva directa o indirecta: tragaluces e invernaderos adosados, se logró incrementar las temperaturas del aire en los ambientes interiores de los módulos, $6,8{ }^{\circ} \mathrm{C}$ para $\mathrm{M} 1$ y $11,2{ }^{\circ} \mathrm{C}$ para $\mathrm{M} 2$.

Journal TECNIA Vol.31 N¹ January-June 2021 
M. Ponce et al.

- La técnica bioclimática que mayor aporta al incremento de temperatura para mejorar el bienestar térmico interior del módulo según las diferentes configuraciones y modificaciones realizadas en las envolventes de $\mathrm{M}_{1}$ y $\mathrm{M} 2$, para las condiciones climáticas de Imata, es el invernadero adosado a $\mathrm{M}_{2}$, con un incremento de temperatura de $5,16{ }^{\circ} \mathrm{C}$ respecto de $\mathrm{M} 1$, el cual se corrobora mediante validación con los resultados de la simulación obteniéndose buenos acuerdos.

- La configuración que presenta un mayor ahorro energético en la demanda de calefacción para mantener la temperatura de diseño del aire interior constante a $15{ }^{\circ} \mathrm{C}$, es cuando la puerta y ventana se mantienen abiertas de 8:00 a 17:00 horas, requiriéndose para M1 14,23 kWhdía ${ }^{-1}$ y para M2 10,29 kWhdía ${ }^{-1}$.

- Finalmente, la mejora del comportamiento térmico se puede atribuir a la mejora significativa del aislamiento térmico y la inclusión de ganancias solares directas e indirectas como las claraboyas e invernadero adosado, este último idóneo para zonas Altoandinas por su buen desempeño y aporte térmico al interior del módulo M2.

\section{AGRADECIMIENTOS}

Este trabajo ha contado con la financiación del Consejo Nacional de Ciencia, Tecnología e Innovación (CONCYTEC), por medio del convenio 024-2016FONDECYT y el contrato 04-2018-FONDECYT BM-IADTMU. J. Molina Agradece a CONCYTEC/FONDECYT/BM por el financiamiento a través del contrato no. 05-2018FONDECYT/BM.

\section{REFERENCIAS}

[1] SENAMHI (2003, Enero). ATLAS DE ENERGÍA SOLAR DEL PERÚ [Online]. Available: https://hdl.handle.net/20.500.12542/343.

[2] Presidencia del Consejo de Ministros (2020). Plan Multisectorial Ante Heladas Y Friaje 2019 - 2021 [Online]. Available: https://www.gob.pe/institucion/pcm/campañas/56-planmultisectorial-ante-heladas-y-friaje-2019-2021.

[3] INEI (2017). Resultados Definitivos de los Censos Nacionales 2017
[Online]. Available: http://censo2017.inei.gob.pe/resultadosdefinitivos-de-los-censos-nacionales-2017/.

[4] INDECI (2017). Instituto Nacional de Defensa Civil - INDECI | Gobierno del Perú [Online]. Available: https://www.gob.pe/indeci.

[5] Canal N (2018, Julio 10). Defensoría: Nueve regiones registran 182 fallecidos a causa de heladas y friaje [Online]. Available: https://canaln.pe/actualidad/defensoria-nueve-regiones-hay-182-

fallecidos-causa-heladas-y-friaje-n329573.

[6] A. Flores, "Construccion de una vivienda solar en base a las propiedades termofísicas y evaluación experimental de su confort térmico en llave", Rev. Investig. Altoandina, vol. 16, no. 1, pp. 177-186, 2014

[7] R. Espinoza et al., "Evaluación experimental de cambios constructivos para lograr confort térmico en una vivienda Altoandina del Perú", Rev. Avances en Energías Renovables y Medio ambiente, vol. 13, pp. 203-210, 2009.

[8] El Peruano (2014). Norma EM. 110 Confort Térmico y Lumínico con Eficiencia Energética [Online]. Available: http://www.construccion.org/normas/rne2012/rne2006.htm.

[9] GIZ-MVCS (2015). Abrigando hogares - Experiencias con medidas de confort térmico en viviendas rurales Altoandinas [Online]. Available:

https://issuu.com/melissakatherynpalma/docs/abrigand o-hogares

[10] J. Molina, "Uso del programa Energyplus para la simulación del consumo energético de un módulo bioclimático ubicado en Imata, Arequipa a 4519 msnm", Tesis de grado, Facultad de Ciencias, UNI, Lima, 2018.

[11] J. Molina, M. Ponce, M. Horn and M. Gómez, "Towards a sustainable bioclimatic approach for the Peruvian high Andean rural area: Evaluation of the thermal contribution of a greenhouse attached to a dwelling", Proc. ISES Sol. World Congr. 2019 IEA SHC Int. Conf. Sol. Heat. Cool. Build. Ind. 2019, pp. 355-364, nov. 2019.

[12] J. Molina, G. Lefebvre, M. Horn and M. M. Gómez, "Design of an experimental bioclimatic module obtained from the analysis of thermal simulations for the community of Imata (4519 m a.s.l.), located in Arequipa, Perú", Inf. Tecnol., vol. 31, no. 2, pp. 173-185, abr. 2020.

[13] SENAMHI (2020). Datos Hidrometeorológicos 2020 [Online]. Available: https://www.senamhi.gob.pe/?p=pronosticometeorologico.

[14] SOLCAST (2020). Histórico y TMY - Datos y especificaciones 2020 [Online]. Available: https://solcast.com/.

Los artículos publicados por TECNIA pueden ser compartidos a través de la licencia Creative Commons: CC BY 4.0. Permisos lejos de este alcance pueden ser consultados a través del correo revistas@uni.edu.pe 